\title{
The genetic landscape of animal behavior
}

\section{Affiliation:}

12

\author{
${ }^{1}$ Department of Biology, Stanford University, Stanford CA, 94305, USA
}

13 
Although most animal behaviors are associated with some form of heritable genetic variation we do not yet understand how genes sculpt behavior across evolution, either directly or indirectly. To address this, I here compile a dataset comprised of over 1,000 genomic loci representing a spectrum of behavioral variation across animal taxa. Comparative analyses reveal that courtship and feeding behaviors are associated with genomic regions of significantly greater effect than other traits, on average three fold greater than other behaviors. Investigations of whole-genome sequencing and phenotypic data for 87 behavioral traits from the Drosophila Genetics Reference Panel indicate that courtship and feeding behaviors have significantly greater genetic contributions and that, in general, behavioral traits overlap little in individual base pairs but increasingly interact at the levels of genes and traits. These results provide evidence that different types of behavior are associated with variable genetic bases and suggest that, across animal evolution, the genetic landscape of behavior is more rugged, yet predictable, than previously thought. 


\section{Introduction}

Nearly all behaviors are associated with some form of heritable genetic variation (Kendler and Greenspan 2006). This interplay between genetic and other forces that shape behavior is complex and disentangling it occupies an array of research endeavors, spanning disciplines from evolutionary biology to psychiatry. Accordingly, recent years have seen reasonable progress toward understanding the genetic architecture of certain behavioral traits using model systems (Reaume and Sokolowski 2011). The general conclusion from this research in mice, flies, worms, and humans is that the genetic architectures of behaviors generally fit an exponential distribution, with a small number of loci of moderate to large effect and a larger number of loci with small effects (Robertson 1967; Flint and Mackay 2009). However, owing to limits in data and methods, the extent to which genetic architectures vary across a full spectrum of behaviors and animal taxa has remained largely unexplored.

Behaviors can exhibit considerable variation in genetic influence. Comparative analyses reveal that behaviors vary substantially in heritability estimates, most often ranging between $10 \%$ and $50 \%$ (Kendler and Greenspan 2006; Mousseau and Roff 1987; Meffert et al. 2002). Analyses of individual behaviors reveal even greater diversity. For example, a single retro-element is responsible variation in a courtship song between Drosophila species (Ding et al. 2016) while other traits, such as deer mouse burrowing, have modular genetic architectures comprised of multiple interacting loci (Weber et al. 2013). Furthermore, the structure and effect of genetic architectures may vary with behavioral traits, as suggested by the preponderance of large effect loci found for insect courtship traits across multiple species (Arbuthnott 2009). Despite these observations the extent to which behavioral traits may systematically vary across species and behaviors remains unknown. Understanding this could provide insights into how behaviors respond to evolutionary processes, the prospects for finding general principles in the genetic evolution of behavior, and even potentially why there has been such variable success in the mapping of human neuropsychiatric traits.

Here, using reports associating behavioral variation with the genes for specific traits across diverse species, I assemble a comparative behavior genetics resource composed of 1,007 significant 
genomic loci from 114 QTL studies conducted in 30 species across 5 taxonomic classes. These data exploit advances in sequencing and genetic marker design that have accelerated reports using quantitative trait locus (QTL) mapping to identify genomic regions that are associated with behavioral variation (Lander and Botstein 1989; Flint and Mackay 2009). With the compiled dataset I compare the genetic architecture of behavioral types across animal taxa. I then corroborate these observations and assay genetic processes involved in the early stages of behavioral differentiation in a natural population using whole genome data from the Drosophila Genetic Resource Panel (DGRP). These analyses provide insight into the genetic architecture of behavior across animals and the interplay between specific behavioral traits and their genetic influence through evolutionary history.

\section{Results and Discussion}

I performed a comprehensive analysis of results aggregated from 114 QTL studies conducted in 30 species across 5 taxonomic classes to assemble a comparative behavior genetics resource composed of 1,007 significant genomic loci (Database S1). The species examined represent over 500 million years of evolutionary divergence and over a broad spectrum of phylogenetic data (Fig 1a). For each locus I annotated the trait measured and its associated effect size (percent phenotypic variation explained), the reported measure of significance (e.g., LOD score), genomic locus, and study sample size. I focused the analyses on the reported effect sizes to allow comparison of the genomic architecture of traits across studies similar to previous meta-analyses of behavioral QTL in mice and flies (Flint 2003; Flint and Mackay 2009).

I found that the distribution of effect sizes in the dataset is similar to that found in these previous studies (Fig 1b). In the majority of loci $(89.51 \%)$ the effect sizes are less than $20 \%$ with a mean effect size of $9.54 \%$, suggesting that the genetic bases of most behaviors assayed are complex and composed of many loci of moderate effect.

Though these results support a model of many loci with small effects for behavior overall, I then asked whether genetic architecture might vary across types of behavior. I identified ten behavioral categories for which traits had been measured in at least two species (See supplementary methods). My 
null hypothesis was that individual categories would likely reflect the overall distribution seen across the dataset, consistent with previous observations that QTL have relatively similar effect sizes across mouse and fly phenotypes (Flint 2003; Flint and Mackay 2009). Surprisingly, I found instead that behaviors differed significantly in their effect sizes. Specifically, loci associated with courtship $(n=124)$ explained significantly more phenotypic variance than all other behaviors combined (Kruskal-Wallis $\mathrm{p}=6.7 \times 10^{-29}$ ) and had a mean effect size three times larger than found in all other categories (Fig. 1c). Loci associated with feeding behaviors $(\mathrm{n}=11)$ also explained significantly more phenotypic variance than all other behaviors combined $\left(\mathrm{p}=6.8 \times 10^{-13}\right)$ while emotion and social behaviors explained significantly less $(\mathrm{p}=$ $8.6 \times 10^{-33} ; \mathrm{p}=2.5 \times 10^{-21}$, respectively). These data suggest that, across species, courtship and feeding behaviors possess genetic architectures different from those of other traits.

\section{Figure 1:}

A

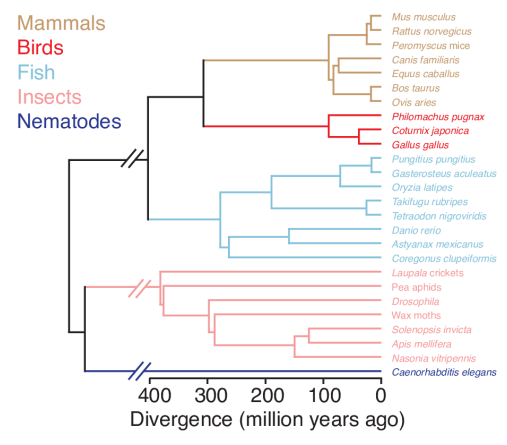

\section{C}

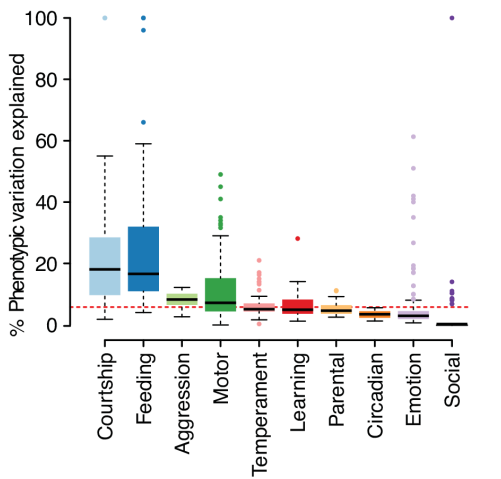

B
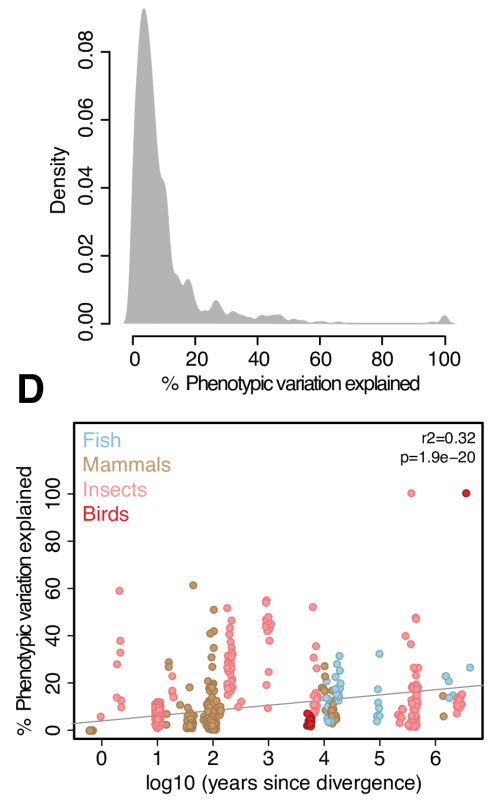

To assess whether these observations arose from differences in the behavioral traits, as byproducts or experimental artifacts I controlled for factors that might have contributed bias. I first considered the effect of intraspecific (within species) compared to interspecific (between species) crosses used for the QTL mapping, a known source of influence in QTL studies (Broman 2001). I indeed found 
that experiments employing interspecific crosses identified loci of significantly higher effect $(\mathrm{p}=4.5 \mathrm{x}$ $\left.10^{-5}\right)$. To control for this quantitatively, I estimated phylogenetic divergence and generation times between the crosses used in each of the 115 studies (Supplementary methods). There was a positive correlation between evolutionary divergence and effect size $\left(r^{2}=0.32, p=1.9 \times 10^{-20}\right.$; Fig. 1d; Supplementary methods). I also considered sample size, a well-known source of bias for which, as might be expected, there was a negative correlation with effect size $\left(r^{2}=-0.37, p<0.0001\right)$.

To test the effect of key variables, evolutionary divergence individually, sample size individually, and both combined, I used three linear models (Supplementary methods). Strikingly, the overall structure of the effect size distribution remained largely unaffected after analysis of the residuals from all three models (Fig S1-3; Supplementary methods). In addition, courtship and feeding behaviors had significantly larger effect sizes even after accounting for these potential sources of bias $\left(\mathrm{p}=1.4 \times 10^{-14}\right.$ and $\mathrm{p}=5.7 \times 10^{-7}$, respectively; Fig $\left.2 \mathrm{a}\right)$.

\section{Figure 2:}

A

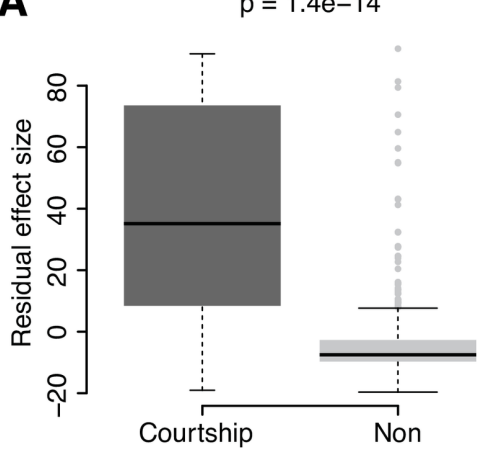

B

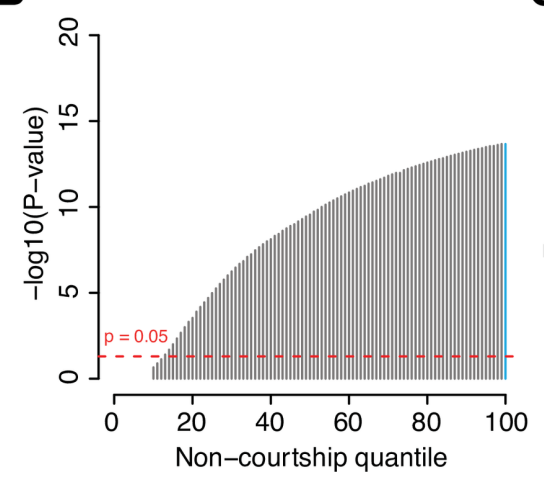

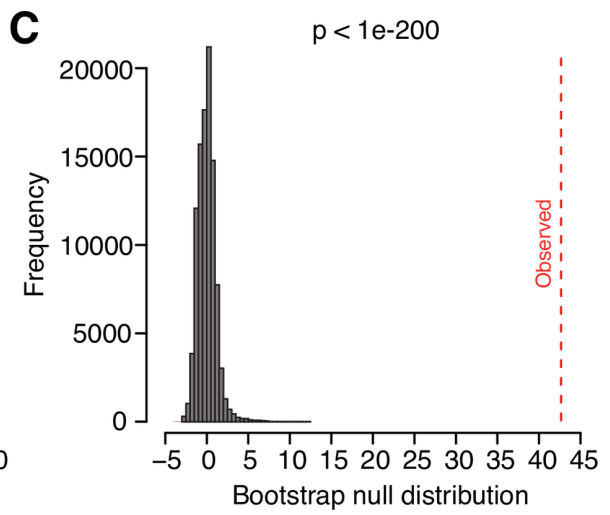

After eliminating sources of potential biases inherent to individual datasets, I next considered the possibility that the detection of courtship and feeding behaviors as outliers was a trivial outcome of our own classification method for grouping single behaviors into ten categories. Minimally assuming that the categorizations of courtship and feeding traits were correct, it is possible that the binning of traits into the other eight categories may have masked a real signal from some biologically relevant categorization. 

categories to the distribution for all other behaviors combined (Supplementary methods). I found that courtship behaviors explained significantly more variation $(\mathrm{p}<0.05)$ than $89 \%$ of non-courtship behaviors while feeding behaviors explained more variation than $46 \%$ of non-feeding behaviors (Fig 2b; Fig S4b). I complemented this test with a bootstrap analysis that created a null distribution from 10,000 permutations of the non-courtship/feeding trait effect sizes. The observed mean adjusted effect size for both courtship and feeding fell significantly outside the bootstrap null distribution created for each comparison $(\mathrm{p}<5 \mathrm{x}$

$1411^{-200}$ )(Fig 2c; Fig S4c). These findings reject the notion that there may be another categorization of noncourtship and feeding behaviors missed by our schema that explains substantially more variation of effect. analyses of the QTL behavior literature in insects found that a majority of courtship traits are associated with few loci of particularly strong effect that play a potential role in rapid speciation through prezygotic isolation (Arbuthnott 2009). In addition, theoretical work has suggested that traits controlling local adaptation during speciation, such as courtship and feeding, evolve more rapidly if they are associated with a smaller number of loci (Gavrilets et al. 2007). Given the importance of behavior's role in the early stages of speciation it may be possible that for the organisms and traits analyzed here, courtship and

151 feeding traits with simpler genetic components of large effect were selected for during the evolution of 152 these lineages. These observations led me to hypothesize that, in a naturally interbreeding population, 153 courtship and feeding behaviors may be associated with more heritable genetic architectures of greater 154 effect when compared to other behavioral traits. comprised of over 200 inbred, fully sequenced Drosophila melanogaster lines isolated from a farmer's

157 market in Raleigh, North Carolina (Mackay et al. 2012). Phenotypic measures for a wide number of 158 behavioral traits are available for the DGRP lines in addition to full genome sequence and variant 159 information, making this resource unique in enabling us to ask larger scale questions about variation and 
160 evolution in behavior. I collected phenotypic measures for 87 behavioral traits spanning 8 categories,

161 produced in 9 separate GWA studies (Jordan et al. 2012; Weber et al. 2012; Swarup et al. 2013; Arya et

162 al. 2015; Gaertner et al. 2015; Garlapow et al. 2015; Morozova et al. 2015; Shorter et al. 2015). SNPs could explain more trait variation than by chance in these cases (Fig. 3a; Supplementary methods).

167 The majority of these traits were enriched for involvement in courtship and feeding: $30 \%$ (6/20) were

\section{Figure 3:}
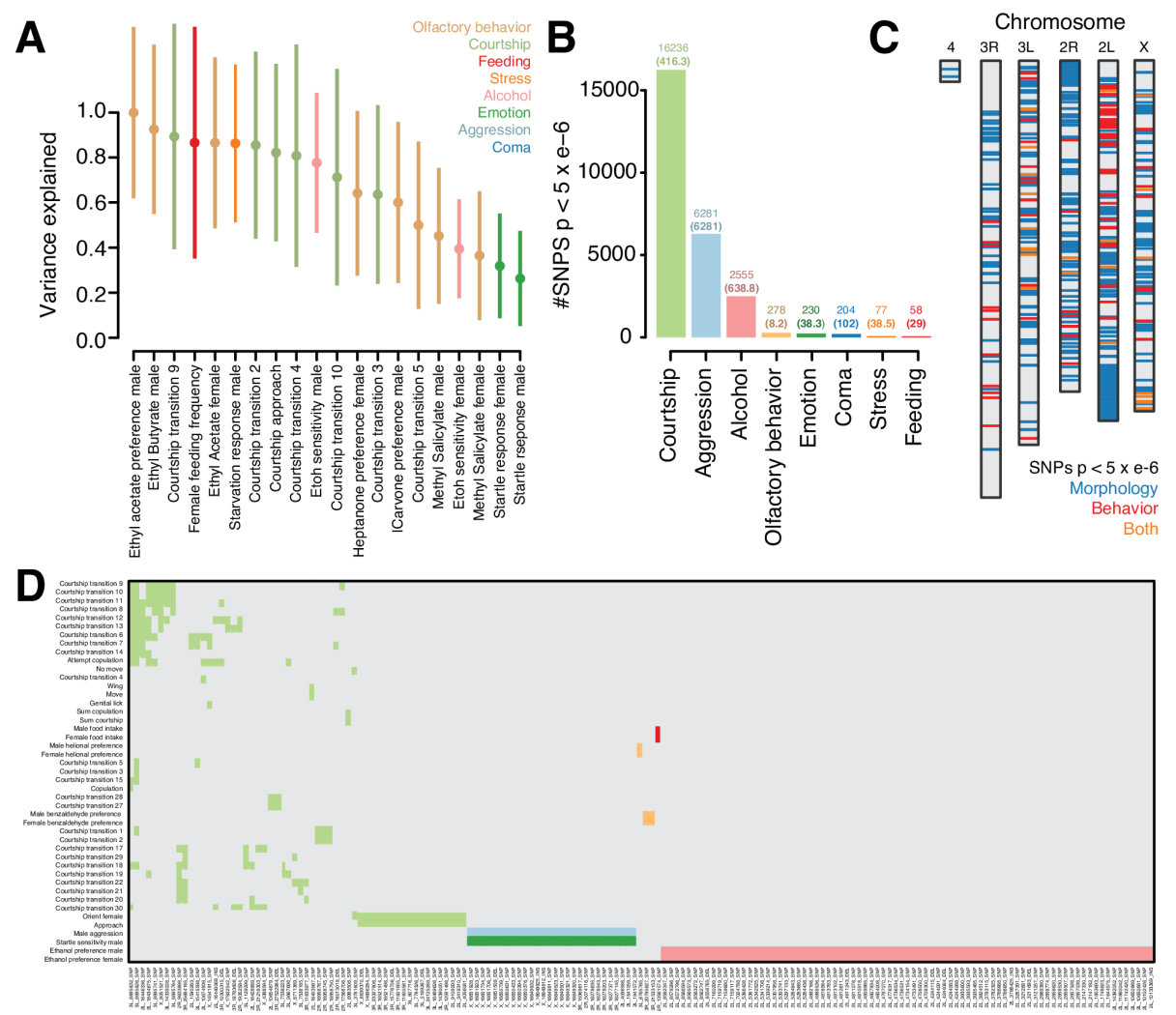
In addition to an increase in genomic heritability, my QTL analyses also showed that the genomic architectures of courtship and feeding traits may be simpler and of higher effect. To test this I performed a separate GWA experiment for each trait across all lines with available phenotypic data and filtered for SNPs with a nominal p-value of $5 \times 10^{-6}$ (Supplementary methods). At this threshold I found 25,919 SNPs (Fig. 3b; Table S1).

I re-ran GCTA for each trait using only SNPs identified at $\mathrm{p}<5 \times 10^{-6}$ from the GWAS (supplementary methods). This test is more conservative compared to genome-wide GCTA since it uses just the fraction of genomic variants significantly associated with each individual trait. After GCTA I found 16 behavioral traits that passed the $\mathrm{p}$-value threshold of $\mathrm{P}<0.05$. Half of these significant traits were courtship behaviors, including the top four traits with the most variation explained by GWAS SNPs (Fig. S5a). The number of GWAS significant SNPs for these 16 traits varied substantially and was positively correlated with the amount of phenotypic variance explained (Fig. S5b). For traits with more SNPs, significant portions of the variance could be accounted for. For example, 665 SNPs could account for $63.52+/-8.42 \%$ of variation in courtship wing movement, 828 accounted for $68.64+/-6.69 \%$ of genital licking behavior, and 8,013 accounted for $78.45+/-5.97 \%$ of courtship approach behavior. The results from both GCTA tests in the DGRP lines support the hypothesis that courtship and feeding-related behaviors are associated with more heritable genetic architectures of large effect, even within less diverged natural populations.

I next used the DGRP lines to query the extent to which genes or genomic loci may affect multiple behavioral traits (pleiotropy) (Greenspan 2004). I exploited the breadth of phenotypic and genomic data available in the DGRP to empirically address this question at three levels: SNPs, genes, and traits. To allow for comparisons of behavior and other trait types I also conducted GWA for 26 morphological traits reported in Vonesch et al. 2016 (Supplementary methods; Table S2). SNPs found to be associated with morphology and behavior at $\mathrm{p}<5 \times 10^{-6}$ were distributed across the Drosophila melanogaster genome, 80 of which were associated with both behavioral and morphological traits (Fig. $3 c)$. 
With this list of variants I queried which individual SNPs were associated with multiple behavioral categories. I identified 169 SNPs associated with at least two behavioral measures. These variants largely segregated within behavioral categories rather than between categories, suggesting that at the level of individual SNPs these traits may have largely independent genetic architectures amongst the DGRP lines (Fig. 3d). Many of these SNPs fell within the same genomic regions. I found 72 genes had at least 2 SNPs associated with multiple traits, several of which contained a multitude of variants (Fig. S6a). These genes are enriched for involved in biological processes such as Notch signaling, receptor activity, and morphogenesis (Supplementary methods; Table S3). In addition, I found 81 intergenic SNPs that each occurred within $20 \mathrm{~kb}$ of their nearest gene - 26 genes in total - suggesting potential regulatory roles for these SNPs (Fig. S6b).

I then assessed the extent to which behaviorally associated variants may act pleiotropically at the trait level, using the list of 25,919 variants associated with behavior. With this I correlated the effect sizes of trait-associated SNPs with the effect sizes of those same variants across all other traits (following ref. 26). The results of this analysis are summarized in the clustered heatmap in Fig. S7. In general I found extensive correlations between behavioral traits, suggesting widespread pleiotropic genetic effects. I also observed several large clusters of highly correlated traits, suggesting a higher-level structure for phenotypic variation based on trait interactions (labeled 1-4 in Fig. S7). The existence of these apparent clusters suggest that, while behavioral categories in the DGRP overlap little in genomic architecture at the individual variant level, there may be common molecular pathways through which different behavioral traits are altered in a correlated fashion.

Finally, I explored pairs of traits with putative directional relationships given the effect sizes of their associated variants. I avoid calling these relationships causal since, given the existence of extensive epistasis and genetic linkage the DGRP lines, it is difficult to identify individual variants of likely causal effect (Huang et al. 2012). I instead sought to elucidate aspects of a directional relationship by 
(summarized as $P_{1} \leftarrow G \rightarrow P_{2}$ compared to $G \rightarrow P_{l} \rightarrow P_{2}$ ) (Pickrell et al. 2016). In addition to the 87 identified behavioral traits, I included the 26 morphological measures to gather insights into potentially directional relationships between behavior and morphology in the DGRP.

I conducted pairwise tests of each trait at which GWAS variants at the $\mathrm{p}<5 \times 10^{-6}$ level were identified. Using a permutation based test I found 143 trait pairs that showed directionality wherein the correlation of effect sizes was strong and significant in one comparison but not the other (Supplementary methods; Fig. 4a).

Trait pairs identified as significant showed an uneven distribution of potential directional effect

238 (rho=-0.86, $\mathrm{p}=8.6 \times 10^{-8}$; supplementary methods).

\section{Figure 4:}
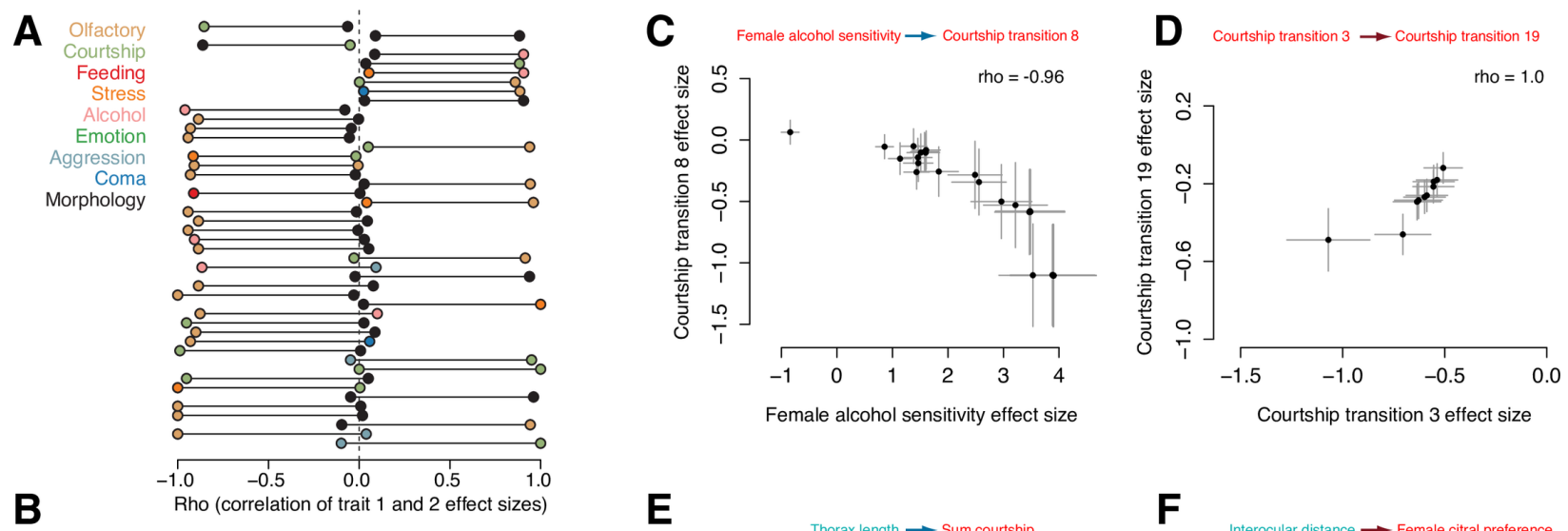

B
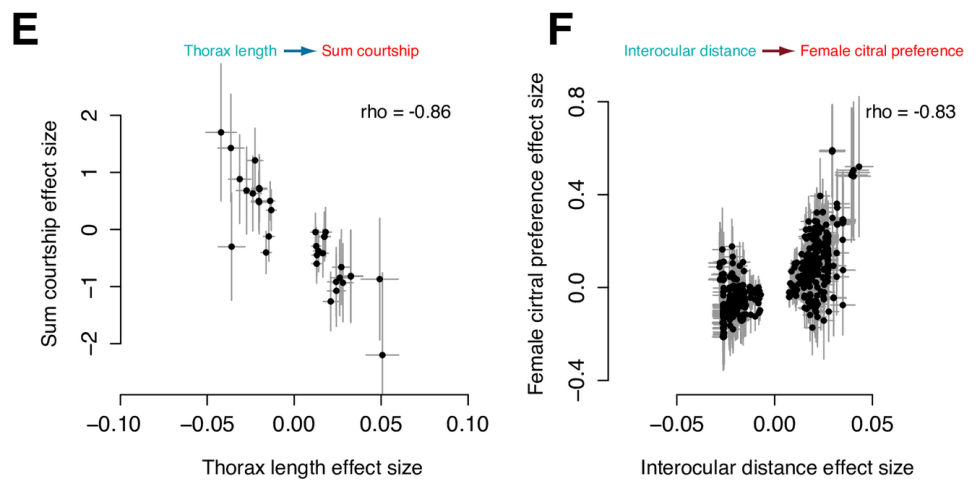
The connection between male courtship behaviors and body size has long been recognized in laboratory strains of Drosophila though with little evidence of a molecular basis for this effect (Ewing 1961). In general I find extensive evidence of both directional $\left(G \rightarrow P_{1} \rightarrow P_{2}\right)$ and general $\left(P_{1} \leftarrow G \rightarrow P_{2}\right)$ pleiotropic 244 effects between traits in the DGRP, supporting the notion that the early stages of behavioral diversification involve the role of genes that can effect multiple types of traits. Furthermore, I observe that while variation in behavior across trait categories is associated with non-overlapping variants these may occur in common genes and molecular pathways with pleiotropic effects, reflecting suggestions of the existence of phenotypic "hotspots" that are recurrently used by evolution to sculpt phenotypes (Stern \& Orgogozo 2008).

Taken together these results suggest that behavioral traits may respond to evolutionary processes with greater variation than previously appreciated. For example, researchers may now anticipate that assaying a courtship ritual will likely yield a higher genetic effect than, say, variation in a personality trait. These insights are supported by observations that behavioral categories vary in their heritability and genomic architecture during even the earliest stages of diversification within populations. Furthermore, such behaviors are associated with a small number of highly pleiotropic genes and these traits interact, indicating that there are identifiable molecular and phenotypic patterns that govern behavior. studies. First, QTL mapping methods possess inherent limitations in detecting the complete genetic architecture of certain traits. For example, QTL studies are often insensitive to the detection of loci with opposing effects on the trait of interest, thus potentially masking important genetic effects from the researcher's analysis (Mackay et al. 2009). Future studies of the genetic architecture of behavior will thus

262 benefit from integrating QTL methods with results from genome-wide sequencing and genetic 263 interrogations directed by genome editing ${ }^{7}$. Second, a more complete survey of behavioral categories 264 within and across a variety of taxa are needed to confidently establish whether or not the patterns 
266 and lab may offer a deeper understanding of the extent to which courtship and feeding behaviors respond

267 uniquely to selective pressures, and which evolutionary and ecological mechanisms may account for this

268 phenomenon. Expanding on this with the tools and data now becoming available, behavioral biology may

269 begin to produce a more nuanced and predictive understanding of the interplay of genetic forces

270 governing the evolution of behavior.

271

272

273

274

275

276

277

278

279 
294 I first identified behavioral QTL through literature search querying online engines (e.g. PUBMED) with 295 the keywords "QTL", "behavior", "quantitative trait locus", and "behavioral". I analyzed the results and collected QTL for each relevant publication identified. In order to gather as many relevant QTL as possible over time I expanded the search to include more specific terms relating to behaviors and categories of interest and to those referenced in previously identified papers. I filtered for loci reported as significant by the original authors, resulting in 1,007 QTL from 115 studies. For each locus I recorded the reported effect size (percent phenotypic variation explained), significance measure, genomic location, sample size, and the number of loci reported overall. QTL studies often report other measures in addition to those that I collected (e.g. broad or narrow sense heritability). While it would be desirable to compare certain of these across behaviors and taxonomic groups I found that, within the studies assayed, the reporting of measures other than those I collected was very inconsistent and allowed for only extremely restricted comparisons. Since the measure used to report significance varied across studies I converted all LOD scores to Log p-values using the following R function (R.C.team 2013):

$$
-\log \left(\operatorname{pchisq}\left(x^{*}(2 * \log (10)), d f=1, \text { lower } \cdot \operatorname{tail}=\text { FALSE }\right) / 2\right)
$$

308 I next classified behaviors following the six groups used in the meta-analysis of mouse QTL studies done 309 by Flint 2003. Several categories represented in our data set were not assayed in this original study (e.g. 310 courtship). In our classification of these I attempted to strike a balance between breadth (to increase the 311 tractability of our comparisons) and biological specificity. To do so I required that a category be 312 represented in at least two species or populations and that the classification match either that reported by 313 the original authors or a reasonable division as reported by the animal behavior literature. The 314 classification of a range of biological traits into broader categories is of course difficult and can 315 repeatedly tempt debate; accordingly this is discussed at length in Flint 2003. I offer that it is important to 316 rigorously test results implicating a broadly defined category as interesting through comparisons of that 317 category to the overall distribution of effects, with the goal of controlling for bias introduced by the 
original classifications (as is discussed below). All QTL and the associated measures mentioned here are

319 available in Table $\mathrm{S} 1$.

320 Phylogeny

321 I used the phylogenetic relationships reported in Ponting 2008 as a template for our phylogeny of species

322 examined (Fig. 1a). I added unrepresented species and adjusted dates of evolutionary divergence using the most recent reports available for each specific clade/species. The following sources were used (along with the associated phylogenetic divergences):

325

326

327

328

329

330

331

332

333

334

335

336

337

338

Ruff/quail and chicken: Jarvis et al. 2014

Quail and chicken: Kayang et al. 2006

Nine spined and three spined stickleback: Guo et al. 2013

Stickleback and teleost: Pfister et al. 2007

Cave fish and teleost divergence: Briggs 2005

Laupala cricket and insect divergence: Misof et al. 2014

Wax moth and insects: Misof et al. 2014

Pea aphids and insects: Misof et al. 2014

Peromyscus and mice/rats: Bedford and Hoekstra 2015

Solenopsis and Apis: Ward 2014

Sheep and cows: Bibi 2013

White fish and teleosts: Betancu-R et al. 2013

\section{Effect size comparisons}

The overall distribution of effect sizes (Fig. 1B) was plotted using the density function in R. Since some behavioral categories possessed relatively small sample sizes all comparisons of effect size were done with the non-parametric Kruskal-Wallis test.

For the analyses plotted in Fig. 2a-2c and Figs. S1-4 I summed the effect sizes of all loci associated with a specific behavioral measure for each study. This was done to allow for a comparison of the maximum amount of phenotypic variance explained for each trait in order to allow for conservative test between courtship and feeding and all other traits. For example, there may have been non-courtship/feeding traits associated with many loci that, on their own, possessed small effects but when added together explained a substantial portion of variation. Following this I filtered for loci where sample size information and 
evolutionary divergence information were available, resulting in 773 loci. The rationales for each estimate of evolutionary divergence are discussed in the next section.

I used several linear models to test for potential biasing effects from evolutionary divergence and sample size, both individually and combined. The resulting residuals from these models are presented in figures S1-3. I found no significant correlations between the residuals from the models and the original variables tested. For the model incorporating sample size alone I observed a correlation between the residuals and sample size itself of $-4.06 \times 10^{-17}$. For the models incorporating evolutionary divergence alone I observed a correlation between the residuals and divergence of $-5.34 \times 10^{-17}$. Finally for the model incorporating both there was a correlation of $-1.16 \times 10^{-16}$ with sample size and a correlation of $3.00 \times 10^{-17}$ for divergence. Given the lack of correlation with either variable this suggests that the combined model successfully controlled for both factors. The residuals for this final combined model were then used for a comparison between all categories (Fig. S1) and between courtship/non-courtship (Fig. 2a) and feeding/non-feeding (Fig. S4a).

For the comparison of the observed courtship and feeding residual effect sizes to the quantiles of all noncourtship/feeding traits I used the following R function(where non_courtship and courtship are vectors of residual effect sizes for these groups):

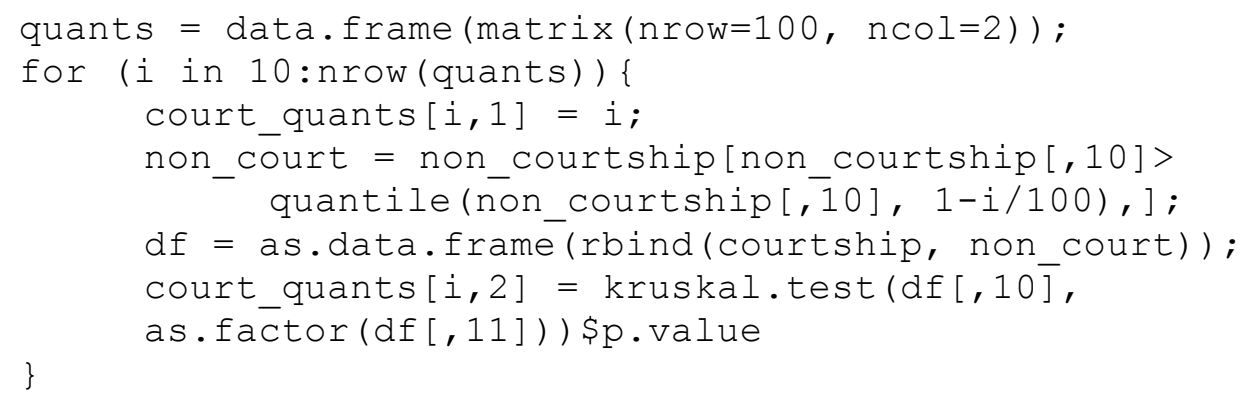

The bootstrap comparisons in Figs. 2c and S3c were done using the custom R function

bootstrap. 2 independent which is available on the Fernald lab website. For these tests I 
permuted the non-courtship/non-feeding residual effect sizes 10,000 times (with replacement) to create a null distribution against I which I tested the observed median residual effect size for each trait. A p-value for each test was calculated by dividing the sum of instances in which the permuted medians were greater than the observed by 10,000 . All plots were produced using base graphics in $\mathrm{R}$ and adjusted for design in Adobe Illustrator.

\section{Data collection of the DGRP lines}

I downloaded the DGRP freeze 2.0 variant calls and plink files from the Drosophila genetics reference panel website (http://dgrp2.gnets.ncsu.edu). Raw data for phenotypic measures were downloaded from the following sources:

$390 \quad$ Starvation resistance: Mackay et al. 2012

391 Startle response: Mackay et al. 2012

392 Chill coma recovery time: Mackay et al. 2012

393 Startle response under oxidative stress: Jordan et al. 2012

394 Negative geotaxis under oxidative stress: Jordan et al. 2012

395 Olfactory behavior (benzaldehyde): Swarup et al. 2013

396 Courtship behavior: Gaertner et al. 2015

397 Olfactory behavior (multiple measures): Arya et al. 2015

398 Aggressive behavior: Shorter et al. 2015

399 Food intake: Garlapow et al. 2015

400 Alcohol sensitivity: Morozova et al. 2015

401 Morphology: Vonesch et al. 2016

402

403 I compiled the raw data into two tables for use in genome-wide analyses of SNP variation, one composed 404 of the 87 behavioral traits obtained and another of the 26 morphological traits. For traits in which multiple 405 measurements were reported I calculated the mean trait measurement and used this for subsequent analyses. I classified traits into behavioral categories in the same fashion as for the evolutionary QTL

407 analyses.

\section{$408 \quad$ Heritability analyses}

409 I first employed genome-wide complex trait analysis (GCTA) to survey genomic heritability across the 87

410 behavioral traits (Yang et al. 2011). For each trait I used greml v1.26.0 to obtain estimates of 411 heritability from genome-wide SNP variation across all DGRP lines for which phenotypic measures were 
available. Using the plink files obtained from the DGRP website (base file name dgrp2) I first created a genotype relatedness matrix for all DGRP lines:

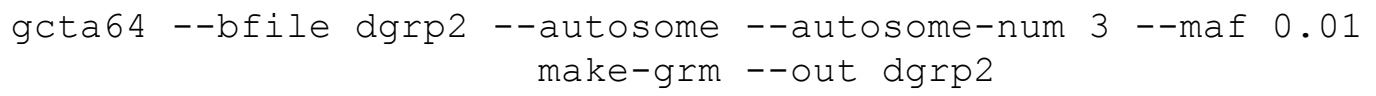

Inidividual phenotype files (*.phen) were created for each trait, including fam and individual IDs and the associate phenotypic measures for each DGRP line. I ran GREML for each phenotype separately:

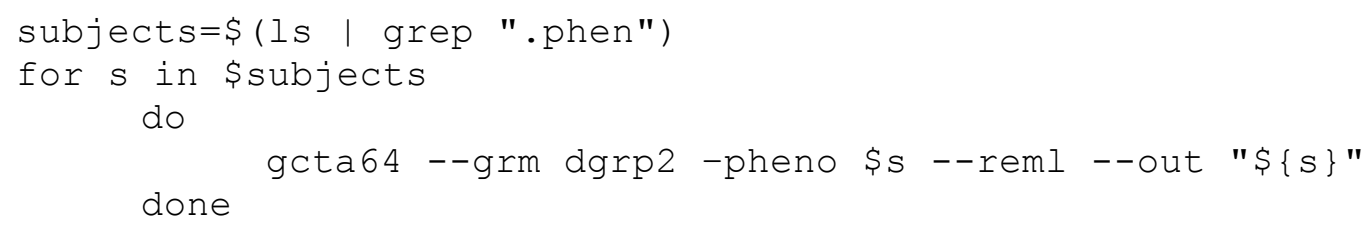

I then filtered for traits in which the reported p-value from GREML was $<0.05$, resulting in 20 traits. Fig 3a. shows the distribution of phenotypic variance explained by genome-wide SNPs as measured by the genotypic variance divided by phenotypic variance $(\mathrm{Vg} / \mathrm{Vp})$.

For the GCTA analyses of just GWAS significant SNPs I compiled a list of associated SNPs for each trait and built a separate genotype relatedness matrix for each by extracting just those SNPs from the plink bed files. I then reran GREML for each trait using the corresponding genotype relatedness matrix and testing only for the SNPs that it contained. Like above I then filtered for traits in which the reported p-value from GREML was $<0.05$, resulting in 16 traits.

\section{Genome-wide association analyses}

The plink and phenotype files from the GCTA analyses were used to conduct separate genome-wide association studies (GWAS) for each trait. I used plink v1.90 to conduct these tests on the combined phenotype matrix ("dgrp_phenos.txt"):

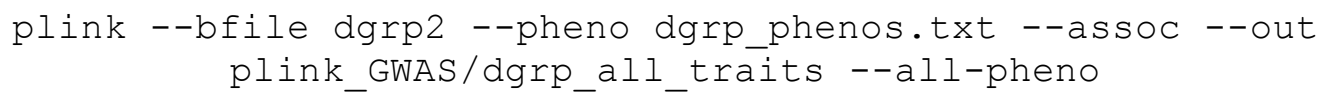

Associations were then filtered for a p-value $<5 \times 10^{-6}$. SNPs associated with multiple traits were identified and plotted using a binary heatmap with the heatmap2 function in R. Genes associated with multiple SNPs were identified using the variant annotation file available on the DGRP website. 
444 I next assayed relationships between SNPs and multiple traits using the effect sizes (betas) in the $445 *$. qassoc files outputted by plink. To do so I compiled a matrix of the effect sizes for all traits at each of the 25,919 significant SNPs (Table S4). This matrix could then be directly queried for comparison of the effect sizes associated with a certain set of SNPs across traits of interest. In order to assess the overall structure of this data set I used Spearman rank correlations to test the associations between all possible trait pairs. The results of this test were visualized using the clustering functionality of heatmap2 in R (Fig. S7).

\section{Tests for trait pair directionality}

452 Directionality in the relationships between trait pairs was tested by first obtaining pairwise rank 453 correlations for each trait pair in which both traits were associated with $>3$ significant SNPs (60 traits).

454 For traits $\mathrm{x}$ and $\mathrm{y}, \mathrm{s}_{1}$ is the vector of SNPs significantly associated with trait $\mathrm{x}$ and $\mathrm{s}_{2}$ is the vector of SNPs 455 significantly associated with trait $\mathrm{y} . \mathrm{xx}$ is the vector of effect sizes at $\mathrm{s}_{1}$ for trait $\mathrm{x}$ and $\mathrm{xy}$ is the vector of effect sizes at $s_{1}$ for trait $y$. Similarily $y y$ is the vector of effect sizes at $s_{2}$ for trait $y$ and $y x$ is the vector of effect sizes at $\mathrm{s}_{2}$ for trait $\mathrm{x}$. Rank correlations can then be obtained for each in $\mathrm{R}$ :

$$
\begin{aligned}
& x_{\text {_cor }}=\operatorname{cor}(\mathrm{xx}, \mathrm{xy}, \text { method="spearman") } \\
& y_{\text {_cor }}=\operatorname{cor}(y y, \mathrm{yx}, \text { method="spearman") }
\end{aligned}
$$

460 Since the strongest signal of directionality would be cases in which the absolute value of $x_{-}$cor/y_cor

461 equals 1 and the other is equal 0 , I assessed directionality as a function of how close to 1 the absolute 462 difference between the correlations was:

I filtered for trait pairs in which rho for one correlation was $>0.5$ and for the other was $<0.1$. I then tested

465 the directional significance of each trait pair by permuting $x x, x y, y y$, and $y x 1,000$ times and 466 recomputed $x_{-}$cor, $y_{-}$Cor, and $\mathrm{D}$ for each permutation. I then calculated a p-value for each trait pair 467 by comparing the vector of permuted $D$ values (pseudo) to the observed $D$ : 


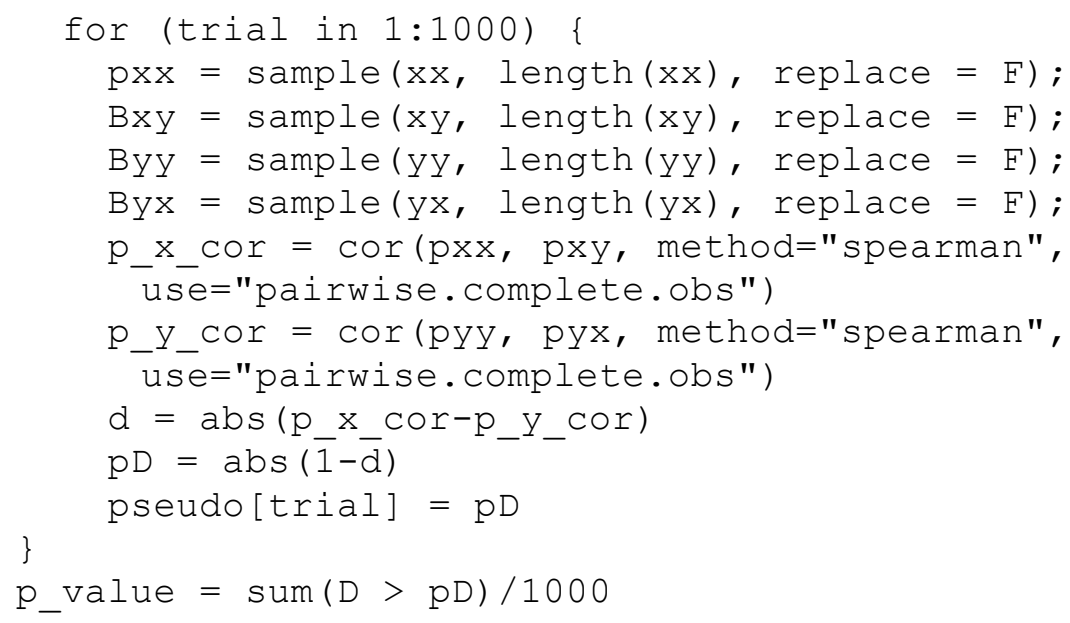

482

483

484 The resulting p-values were adjusted using Bonferroni correction.

485

486

487

488

489

490

491

492

493

494

495

496

497 
498 Acknowledgments: I thank Hunter Fraser, Russell Fernald, Christopher Martin, Graham Coop, David

499 Kingsley, Austin Hilliard, Trudy Mackay, and David Stern for critical reading and useful discussions.

500

501

502

503

504

505

506

507

508

509

510

511

512

513

514

515

516

517

518 
Fig. 1. The genomic landscape of animal behavior $\mid$ (A) Phylogeny of all species studied in which genomic loci were collected for the meta-analysis. (B) Density plot of the distribution of effect sizes for all behavioral traits studied. (C) Boxplot of effect sizes (\% variation explained) by behavioral category. since divergence) and effect size.

Fig. 2 Assaying the genetic architecture of courtship | (A) Boxplot of the comparison between the residual effect sizes of courtship and non-courtship behaviors that resulted from a linear model controlling for sample size and evolutionary divergence. (B) Quantile-based - $\log 10(\mathrm{p}$-values) comparing the residual effect sizes of courtship and non-courtship behaviors at each quantile cutoff. The blue line corresponds to the comparison in Fig 2a. (C) The null distribution resulting from bootstrapping all non-courtship residual effect sizes for 10,000 permutations and the observed median residual effect size for courtship (dashed red line). Heritability estimates $(\mathrm{V}(\mathrm{G}) / \mathrm{Vp}$ ) from GCTA for the 20 measures identified as significant (p-value < 0.05), colored by behavioral category. (B) Barplot summarizing the number of SNPs with $\mathrm{p}<5 \times 10^{-6}$ collected for each behavioral category from GWAS on 87 traits. (C) The distribution of SNPs with $\mathrm{p}<5$ x $10^{-6}$ across the Drosophila melanogaster genome for morphological (blue) behavioral traits (red) and SNPs that associate with measures of both (orange) (D) Heatmap representing the distribution of shared SNPs with $\mathrm{p}<5 \times 10^{-6}$ across all behavioral traits. Plotted are SNPs that possess associations with at least two behavioral traits, colored by the categories highlighted in (A).

540 identified as significant by permutation testing. Plotted are traits where the significant correlation

541 possesses a rho $>0.85$. The significant correlation is represented by a red circle. (B) Barplot summarizing

542 the number of significant trait pairs identified where the focal trait is either behavioral or morphological 543 with a correlation one of these two domains. Behavioral focal traits are colored red, morphological traits 
544 are colored blue. (C-F) Scatterplots of the effect sizes for the focal SNPs of example significant trait

545 pairs. Standard errors are plotted as grey lines. Positive correlations are represented by red arrows and

546 negative correlations are represented by blue arrows.

547

548

549

550

551

552

553

554

555

556

557

558

559

560

561

562

563

564

565

566

567

568

569 


\section{References:}

1. Arbuthnott D. The genetic architecture of insect courtship behavior and premating isolation. Heredity (Edinb). 2009;103(1):15-22. doi: 10.1038/hdy.2009.22. PubMed PMID: 19259113. 2. Arya GH, Magwire MM, Huang W, Serrano-Negron YL, Mackay TF, Anholt RR. The genetic basis for variation in olfactory behavior in Drosophila melanogaster. Chem Senses. 2015;40(4):23343. doi: 10.1093/chemse/bjv001. PubMed PMID: 25687947; PubMed Central PMCID: PMCPMC4398050.

3. Bedford NL, Hoekstra HE. Peromyscus mice as a model for studying natural variation. Elife. 2015;4. doi: 10.7554/eLife.06813. PubMed PMID: 26083802; PubMed Central PMCID: PMCPMC4470249.

4. Betancur RR, Broughton RE, Wiley EO, Carpenter K, Lopez JA, Li C, et al. The tree of life and a new classification of bony fishes. PLoS Curr. 2013;5. doi:

10.1371/currents.tol.53ba26640df0ccaee75bb165c8c26288. PubMed PMID: 23653398; PubMed Central PMCID: PMCPMC3644299.

5. Bibi F. A multi-calibrated mitochondrial phylogeny of extant Bovidae (Artiodactyla, Ruminantia) and the importance of the fossil record to systematics. BMC Evolutionary Biology. 2013;13;166. doi: 10.1186/1471-2148-13-166. PubMed PMID: 23927069; PubMed Central PMCID: PMC3751017. 6. Briggs JC. The biogeography of otophysan fishes (Ostariophysi : Otophysi): a new appraisal. (vol 32,pg 287, 2005). J Biogeogr. 2005;32(5):919-. doi: 10.1111/j.1365-2699.2005.01286.x. PubMed PMID: WOS:000228751400015.

7. Broman KW. Review of statistical methods for QTL mapping in experimental crosses. Lab Anim (NY). 2001;30(7):44-52. PubMed PMID: 11469113.

8. Ding Y, Berrocal A, Morita T, Longden KD, Stern DL. Natural courtship song variation caused by an intronic retroelement in an ion channel gene. Nature. 2016;536(7616):329-32. doi:

10.1038/nature19093. PubMed PMID: 27509856. 
9. Ewing AW. Body size and courtship behaviour in Drosophila melanogaster. Animal Behaviour. 1961;9:93-9.

10. Flint J, Mackay TF. Genetic architecture of quantitative traits in mice, flies, and humans. Genome Res. 2009;19(5):723-33. doi: 10.1101/gr.086660.108. PubMed PMID: 19411597; PubMed Central PMCID: PMCPMC3647534.

11. Flint J. Analysis of quantitative trait loci that influence animal behavior. J Neurobiol. 2003;54(1):46-77. doi: 10.1002/neu.10161. PubMed PMID: 12486698.

12. Gaertner BE, Ruedi EA, McCoy LJ, Moore JM, Wolfner MF, Mackay TF. Heritable variation in courtship patterns in Drosophila melanogaster. G3 (Bethesda). 2015;5(4):531-9. doi:

10.1534/g3.114.014811. PubMed PMID: 25650358; PubMed Central PMCID: PMCPMC4390569. 13. Garlapow ME, Huang W, Yarboro MT, Peterson KR, Mackay TF. Quantitative Genetics of Food Intake in Drosophila melanogaster. Plos One. 2015;10(9):e0138129. doi:

10.1371/journal.pone.0138129. PubMed PMID: 26375667; PubMed Central PMCID: PMCPMC4574202.

14. Gavrilets S, Vose A, Barluenga M, Salzburger W, Meyer A. Case studies and mathematical models of ecological speciation. 1. Cichlids in a crater lake. Mol Ecol. 2007;16(14):2893-909. doi: 10.1111/j.1365-294X.2007.03305.x. PubMed PMID: 17614905.

15. Greenspan RJ. E pluribus unum, ex uno plura: quantitative and single-gene perspectives on the study of behavior. Annu Rev Neurosci. 2004;27:79-105. doi:

10.1146/annurev.neuro.27.070203.144323. PubMed PMID: 15217327.

16. Guo B, Chain FJ, Bornberg-Bauer E, Leder EH, Merila J. Genomic divergence between nine- and three-spined sticklebacks. BMC Genomics. 2013;14:756. doi: 10.1186/1471-2164-14-756. PubMed PMID: 24188282; PubMed Central PMCID: PMCPMC4046692.

17. Huang W, Richards S, Carbone MA, Zhu D, Anholt RR, Ayroles JF, et al. Epistasis dominates the genetic architecture of Drosophila quantitative traits. Proc Natl Acad Sci U S A. 2012;109(39):15553- 
9. doi: 10.1073/pnas.1213423109. PubMed PMID: 22949659; PubMed Central PMCID: PMCPMC3465439.

18. Jarvis ED, Mirarab S, Aberer AJ, Li B, Houde P, Li C, et al. Whole-genome analyses resolve early branches in the tree of life of modern birds. Science. 2014;346(6215):1320-31. doi:

10.1126/science.1253451. PubMed PMID: 25504713; PubMed Central PMCID: PMCPMC4405904.

19. Jordan KW, Craver KL, Magwire MM, Cubilla CE, Mackay TF, Anholt RR. Genome-wide association for sensitivity to chronic oxidative stress in Drosophila melanogaster. Plos One. 2012;7(6):e38722. doi: 10.1371/journal.pone.0038722. PubMed PMID: 22715409; PubMed Central PMCID: PMCPMC3371005.

20. Kayang BB, Fillon V, Inoue-Murayama M, Miwa M, Leroux S, Feve K, et al. Integrated maps in quail (Coturnix japonica) confirm the high degree of synteny conservation with chicken (Gallus gallus) despite 35 million years of divergence. BMC Genomics. 2006;7:101. doi: 10.1186/1471-21647-101. PubMed PMID: 16669996; PubMed Central PMCID: PMCPMC1534036.

21. Kendler KS, Greenspan RJ. The nature of genetic influences on behavior: lessons from "simpler" organisms. Am J Psychiatry. 2006;163(10):1683-94. doi: 10.1176/ajp.2006.163.10.1683. PubMed PMID: 17012675.

22. Lander ES, Botstein D. Mapping mendelian factors underlying quantitative traits using RFLP linkage maps. Genetics. 1989;121(1):185-99. PubMed PMID: 2563713; PubMed Central PMCID: PMCPMC1203601.

23. Mackay TF, Richards S, Stone EA, Barbadilla A, Ayroles JF, Zhu D, et al. The Drosophila melanogaster Genetic Reference Panel. Nature. 2012;482(7384):173-8. doi: 10.1038/nature10811. PubMed PMID: 22318601; PubMed Central PMCID: PMCPMC3683990.

24. Mackay TF, Stone EA, Ayroles JF. The genetics of quantitative traits: challenges and prospects. Nat Rev Genet. 2009;10(8):565-77. doi: 10.1038/nrg2612. PubMed PMID: 19584810.

25. Meffert LM, Hicks SK, Regan JL. Nonadditive genetic effects in animal behavior. Am Nat. 2002;160 Suppl 6:S198-213. doi: 10.1086/342896. PubMed PMID: 18707477. 
26. Misof B, Liu S, Meusemann K, Peters RS, Donath A, Mayer C, et al. Phylogenomics resolves the timing and pattern of insect evolution. Science. 2014;346(6210):763-7. doi:

10.1126/science.1257570. PubMed PMID: 25378627.

27. Morozova TV, Huang W, Pray VA, Whitham T, Anholt RR, Mackay TF. Polymorphisms in early neurodevelopmental genes affect natural variation in alcohol sensitivity in adult drosophila. BMC Genomics. 2015;16:865. doi: 10.1186/s12864-015-2064-5. PubMed PMID: 26503115; PubMed Central PMCID: PMCPMC4624176.

28. Mousseau TA, Roff DA. Natural selection and the heritability of fitness components. Heredity (Edinb). 1987;59 ( Pt 2):181-97. PubMed PMID: 3316130.

29. Pfister P, Randall J, Montoya-Burgos JI, Rodriguex I. Divergent evolution among teleost V1r receptor genes. PLoS One. 2007. doi: 10.1371/journal.pone.0000379. PubMed PMID: 17440615; PubMed Central PMCID: PMC1849887.

30. Pickrell JK, Berisa T, Liu JZ, Segurel L, Tung JY, Hinds DA. Detection and interpretation of shared genetic influences on 42 human traits. Nat Genet. 2016;48(7):709-17. doi: 10.1038/ng.3570. PubMed PMID: 27182965.

31. Ponting CP. The functional repertoires of metazoan genomes. Nat Rev Genet. 2008;9(9):689-98. doi: 10.1038/nrg2413. PubMed PMID: 18663365.

32. Reaume CJ, Sokolowski MB. Conservation of gene function in behaviour. Philos T R Soc B. 2011;366(1574):2100-10. doi: 10.1098/rstb.2011.0028. PubMed PMID: WOS:000291784000005. 33. Robertson A. The nature of quantitative genetic variation. In: Brink R.A., Styles E.D., editors. Heritage from Mendel. University of Wisconsin; Madison, WI: 1967. pp. 265-280 34. Shorter J, Couch C, Huang W, Carbone MA, Peiffer J, Anholt RR, et al. Genetic architecture of natural variation in Drosophila melanogaster aggressive behavior. Proc Natl Acad Sci U S A. 2015;112(27):E3555-63. doi: 10.1073/pnas.1510104112. PubMed PMID: 26100892; PubMed Central PMCID: PMCPMC4500262. 
35. Stern DL, Orgogozo V. The loci of evolution: how predictable is genetic evolution? Evolution. 2008;62(9):2155-77. doi: 10.1111/j.1558-5646.2008.00450.x. PubMed PMID: 18616572; PubMed Central PMCID: PMCPMC2613234.

36. Swarup S, Huang W, Mackay TF, Anholt RR. Analysis of natural variation reveals neurogenetic networks for Drosophila olfactory behavior. Proc Natl Acad Sci U S A. 2013;110(3):1017-22. doi: 10.1073/pnas.1220168110. PubMed PMID: 23277560; PubMed Central PMCID: PMCPMC3549129. 37. Team RC. R: A language and environment for statistical computing. Vienna, Austria: R Foundation for Statistical Computing; 2013.

38. Vonesch SC, Lamparter D, Mackay TF, Bergmann S, Hafen E. Genome-Wide Analysis Reveals Novel Regulators of Growth in Drosophila melanogaster. PLoS Genet. 2016;12(1):e1005616. doi: 10.1371/journal.pgen.1005616. PubMed PMID: 26751788; PubMed Central PMCID: PMCPMC4709145.

39. Ward PS. The Phylogeny and Evolution of Ants. Annu Rev Ecol Evol S. 2014;45:23-43. doi: 10.1146/annurev-ecolsys-120213-091824. PubMed PMID: WOS:000348461700002. 40. Weber AL, Khan GF, Magwire MM, Tabor CL, Mackay TF, Anholt RR. Genome-wide association analysis of oxidative stress resistance in Drosophila melanogaster. Plos One. 2012;7(4):e34745. doi: 10.1371/journal.pone.0034745. PubMed PMID: 22496853; PubMed Central PMCID: PMCPMC3319608.

41. Weber JN, Peterson BK, Hoekstra HE. Discrete genetic modules are responsible for complex burrow evolution in Peromyscus mice. Nature. 2013;493(7432):402-5. doi: 10.1038/nature11816. PubMed PMID: 23325221.

42. Yang J, Lee SH, Goddard ME, Visscher PM. GCTA: a tool for genome-wide complex trait analysis. Am J Hum Genet. 2011;88(1):76-82. doi: 10.1016/j.ajhg.2010.11.011. PubMed PMID: 21167468; PubMed Central PMCID: PMCPMC3014363. 